\title{
Azithromycin nanoparticles: Cyclic voltammetric study in human blood serum samples at electrochemical analysis
}

\author{
Alaa Laebi Abdullah', Muhammed Mizher Radhi², Intesar Nadhum Khelkal', \\ Eman Natiq Naji ${ }^{1}$ \\ ${ }^{1}$ Biology Department, Science College, Mustanserya University, Baghdad, Iraq \\ ${ }^{2}$ Health and Medical Technology College, Baghdad, Iraq
}

\begin{abstract}
In a new study to identify one of the anti-inflammatory, such as azithromycin and its effects on the composition of blood using one of the methods of electrochemical analysis and converted to nanoparticles using cyclic voltammetry. It was found that azithromycin nanoparticles (AZNPs) has oxidation - reduction current peaks in blood medium at 1 and $0.75 \mathrm{~V}$ respectively, these peaks were affected by different $\mathrm{pH}$, the anodic peak was disappeared in alkaline blood medium, while the redox peaks enhanced in acidic blood medium. So, AZNPs acts as anti-oxidant antibiotic in alkaline medium.
\end{abstract}

Keywords: cyclic voltammetry, azithromycin nanoparticles (AZNPs), pH, blood medium

\section{INTRODUCTION}

The special study of blood medium as an electrolyte in electrochemical analysis by cyclic voltammetric has a good results in the oxidation reduction reaction of chemicals and drugs in blood medium [1-5].

Azithromycin is an antibiotic used to treat a number of bacterial infections. This includes middle ear infections, sore throat, pneumonia, traveler diarrhea, and some other intestinal diseases. It can also be used for a number of sexually transmitted diseases, including chlamydia and gonorrhea infection. Along with other drugs, it can also be used for malaria. It can be taken orally or intravenously in doses once a day, Figure 1 shows the structure of the Azithromycin compound [6].

A modified glass-carbon electrode (GCE) coated with a doped carbon nanotubes thin film. This modified electrode displays enhanced efficacy of the oxidation current peak of azithromycin. A method is also described to evaluate the serum azithro- mycin reaction in serum. The electrochemical behavior of azithromycin as well as its interaction with the serum in this nanoparticle film electrode which was investigated by cyclic voltammetry, linear sweep voltammogram measurement, differential pulsed voltammetry and chronocoulometry. Correlation number and persistence of correlation between azithromycin and serum albumin were obtained [7].

A selective and sensitive procedure was developed based on a polymer for the detection and identification of azithromycin antibiotics. Azithromycin / aniline were made with electrolyte (to form polyaniline) on the surface of carbon nano-gold-modified glassy carbon electrode and graphene oxide, where aniline acts as a monomer and azithromycin as a template. The proposed sensor was successfully applied to detect azithromycin in human serum [8].

Recent developments in bio-analysis of pharmaceutical compounds with particular emphasis were 


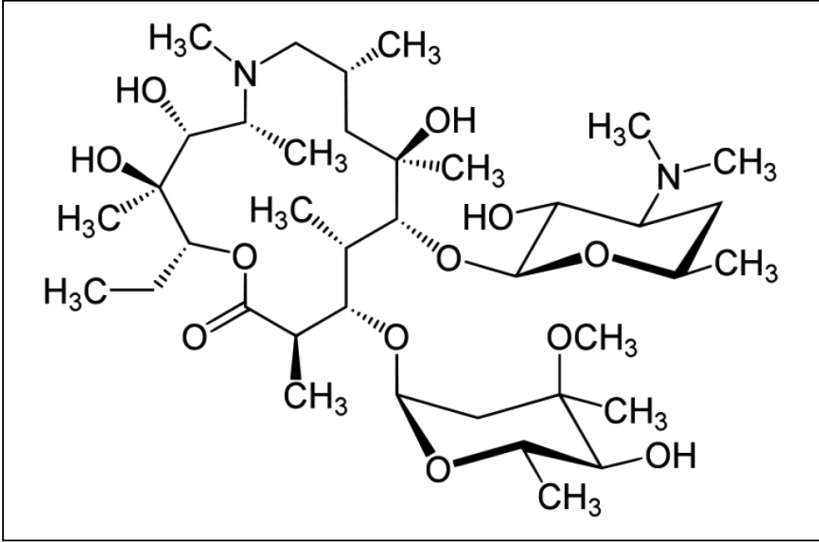

FIGURE 1. The structure of azithromycin

acted with development and application of electrode materials and measurement strategies. The advantages of electrolysis techniques are identified and illustrated as alternatives to other analytical procedures such as rapid response, sensitivity and low detection limits. Particular emphasis is placed on carbon-based materials for voltammetric analysis [9].

\section{EXPERIMENTAL METHOD}

\section{Preparation of azithromycin nanoparticles}

Lyophylization (freezing method) the molecules of azithromycin nanoparticles are also prepared by dissolving $0.75 \mathrm{~g}$ of ceftriaxone micro particles in $150 \mathrm{ml}$ of distilled water. The azithromycin suspension was cooled and the ice crystals of pure water formed at $-18^{\circ} \mathrm{C}$. The second step involves blending the ice from the frozen product by passing thermal air from the Lyophylization tool rack to the frozen solution in the flask, and leaking the flying ice and water vapor through the dried part of the product to the surface of the material. For example, water vapor is transported from the product surface through the chamber to the condenser, and the water vapor condenses on the condenser. At the end of the sublimation step a porous dam is formed. Its pores correspond to the areas occupied by ice crystals. The third step is drying involves removing the absorbed water from the product. All steps must be continuous about 48-72 hours [10].

\section{Materials}

Azithromycin compound form as yellowish powder was bought from Fujian fukang (China) and blood samples were extracted from healthy hu- mans which received from the center medicine of Baghdad City was used in the analysis after separation the serum from the whole blood by Electronic centrifuge type 8-1 (3,000 cycles/min). Deionize water was used for the preparation of aqueous solutions. All the serum of blood samples was diluted with deionize water by ratio $1: 9 \mathrm{ml}$ (serum: deionize water), $10 \mathrm{ml}$ of diluted serum was placed in the cyclic voltammetric cell (volume of $15 \mathrm{ml}$ ).

\section{Apparatus}

\section{Cyclic voltammetric technique}

Instrument series EZstat (Potentiostat / Glvanostat) from NuVant Systems Company (Made in USA). The electrochemical analytical cell was connected with the potentio-status device and monitored through a program that was installed on the PC to conduct periodic voltammetry (CV). Silver electrode contains silver- silver chloride $(\mathrm{Ag} / \mathrm{AgCl}$ in $3 \mathrm{M} \mathrm{KCl}$ ) and platinum wire (diameter $1 \mathrm{~mm}$ ) was used as reference and counter electrodes respectively. The glass working carbon electrode (GCE) was used in this study after cleaning by polishing with an alumina solution and treated with ultrasonic water path for ten minutes for measurement performance.

\section{Lyophylization instrument}

Lyophylization instrument from LABCONCO Company (U.S.A.) was used to preparation of azithromycin nanoparticles from micro particles by deep freezing technique as shown in Figure 2.

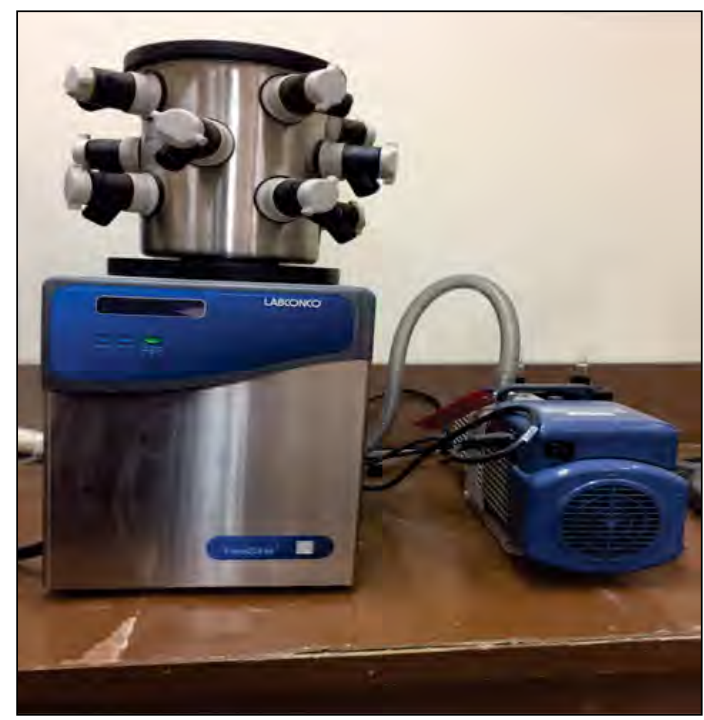

FIGURE 2. Lyophylization instrument, LABCONCO Company (U.S.A.) 


\section{Scanning Electron Microscopy (SEM)}

Figure 3, 4 and 5 are the scanning electron microscopy of the prepared azithromycin nanoparticles (AZNPs) at 200, 500 and $50 \mathrm{~nm}$ respectively, which illustrated the morphology details of the nanoparticles as spherical forms with nano dimeter range $(5-11 \mathrm{~nm})$.

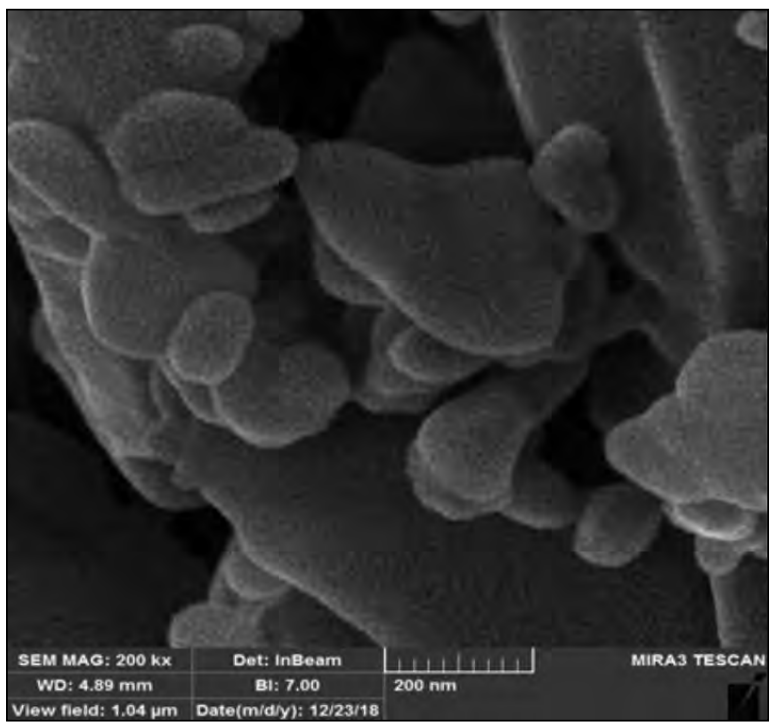

FIGURE 3. SEM of the azithromycin nanoparticles at enlargement of $200 \mathrm{~nm}$

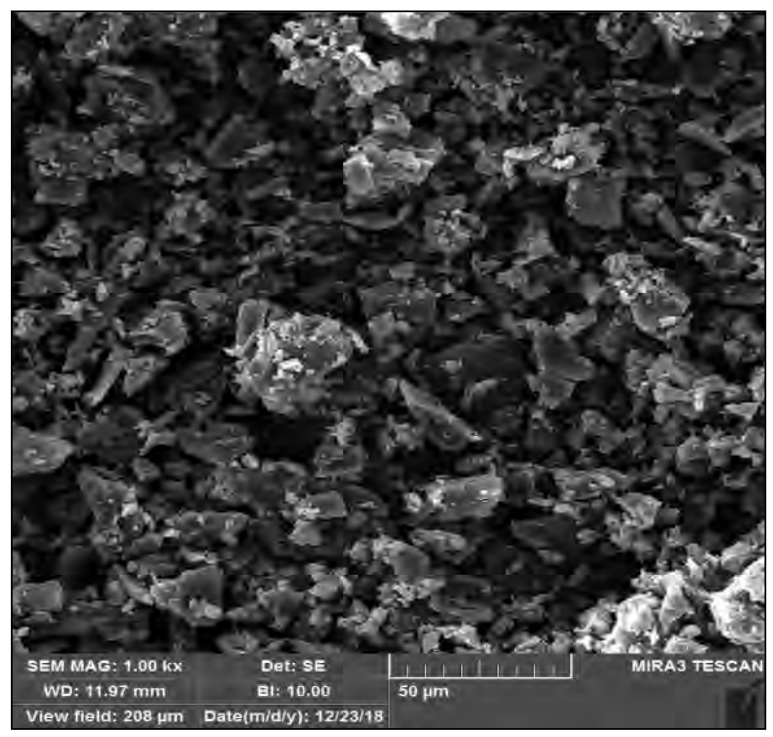

FIGURE 4. SEM of the azithromycin nanoparticles at enlargement of $500 \mathrm{~nm}$

\section{Atomic Force Microscopy (AFM)}

The atomic force microscopy (AFM) technique is good method for identified the dimeter of nanoparticles. The diameter of prepared azithromycin nanoparticles was determined by AFM which found as average diameter $100 \mathrm{~nm}$ as shown in figures 6 , 7 and 8.

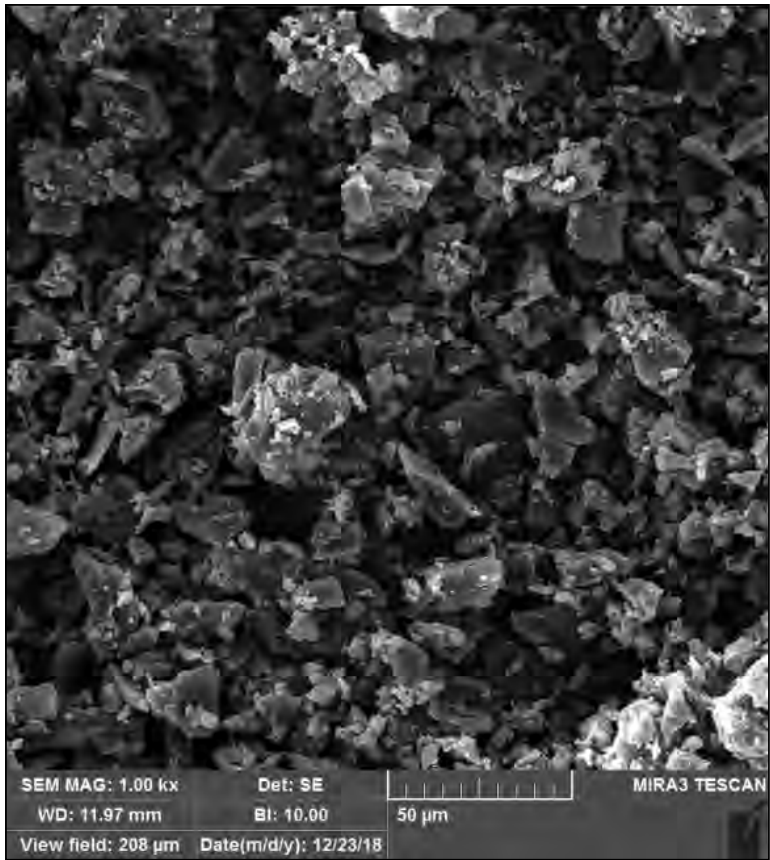

FIGURE 5. SEM of the azithromycin nanoparticles at enlargement of 50 um

\section{RESULTS AND DISCUSSION}

\section{Effect of different concentration of azithromycin nanoparticles in blood serum}

Different concentrations of azithromycin nanoparticles in blood serum were studied using GCE to determine the sensitivity of the nanoparticles in blood medium against to the GCE. Figure 9 shows the cyclic voltammogram of different concentration of azithromycin nanoparticles in blood serum, which enhanced the oxidation-reduction current peaks against to the increasing concentrations of azithromycin nanoparticles in blood medium $[11,12]$.

\section{Effect of different pH on azithromycin nanoparti- cles}

The normal blood medium has a neutral $\mathrm{pH}=7$. But the blood with azithromycin nanoparticles has different properties, figure 10 illustrated that azithromycin nanoparticles has new oxidation current peak at $0.75 \mathrm{~V}$ in acidic medium $\mathrm{pH}=5$, and disappearing the oxidation one in alkaline $\mathrm{pH}=11$ and enhanced the reduction peak at $-0.75 \mathrm{~V}$ [13].

So the azithromycin nanoparticles act as anti-oxidative antibiotic reagent in alkaline blood medium as shown in figure 10. Figure 11 and 12 explained the effect of oxidation and reduction 


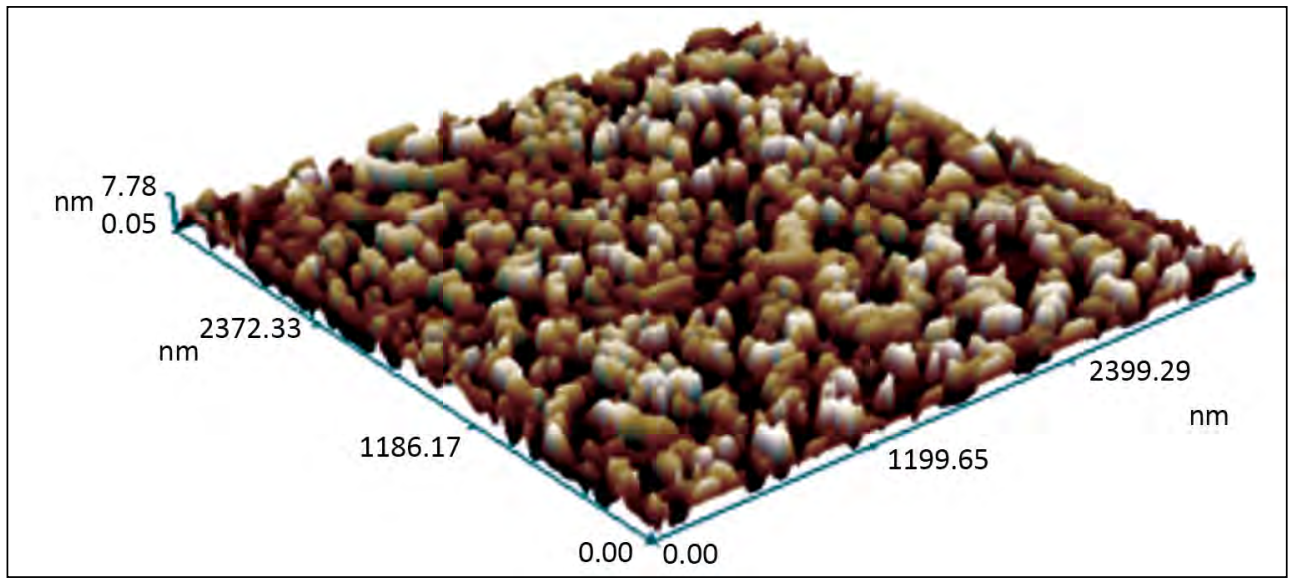

FIGURE 6. AFM of the azithromycin nanoparticles

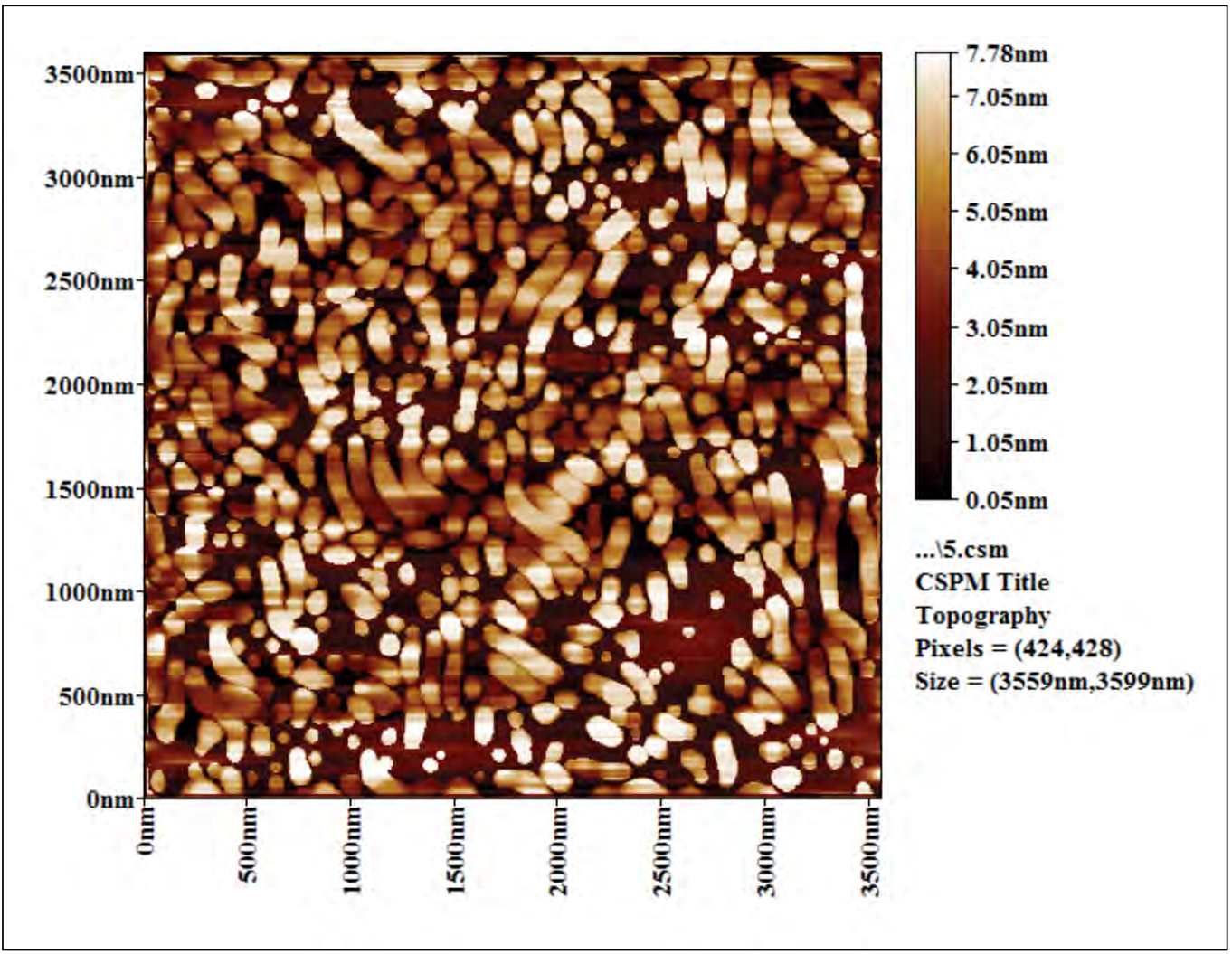

FIGURE 7. AFM of the azithromycin nanoparticles

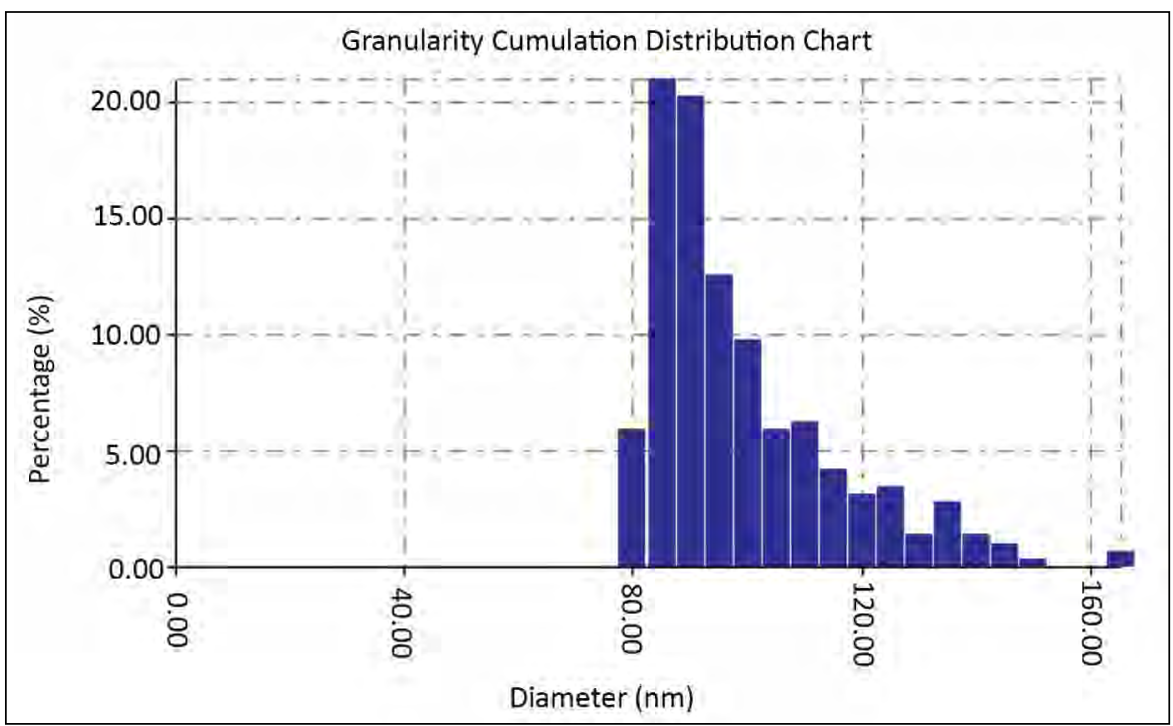

FIGURE 8. Cumulative distribution diagram of azithromycin nanoparticles diameter 


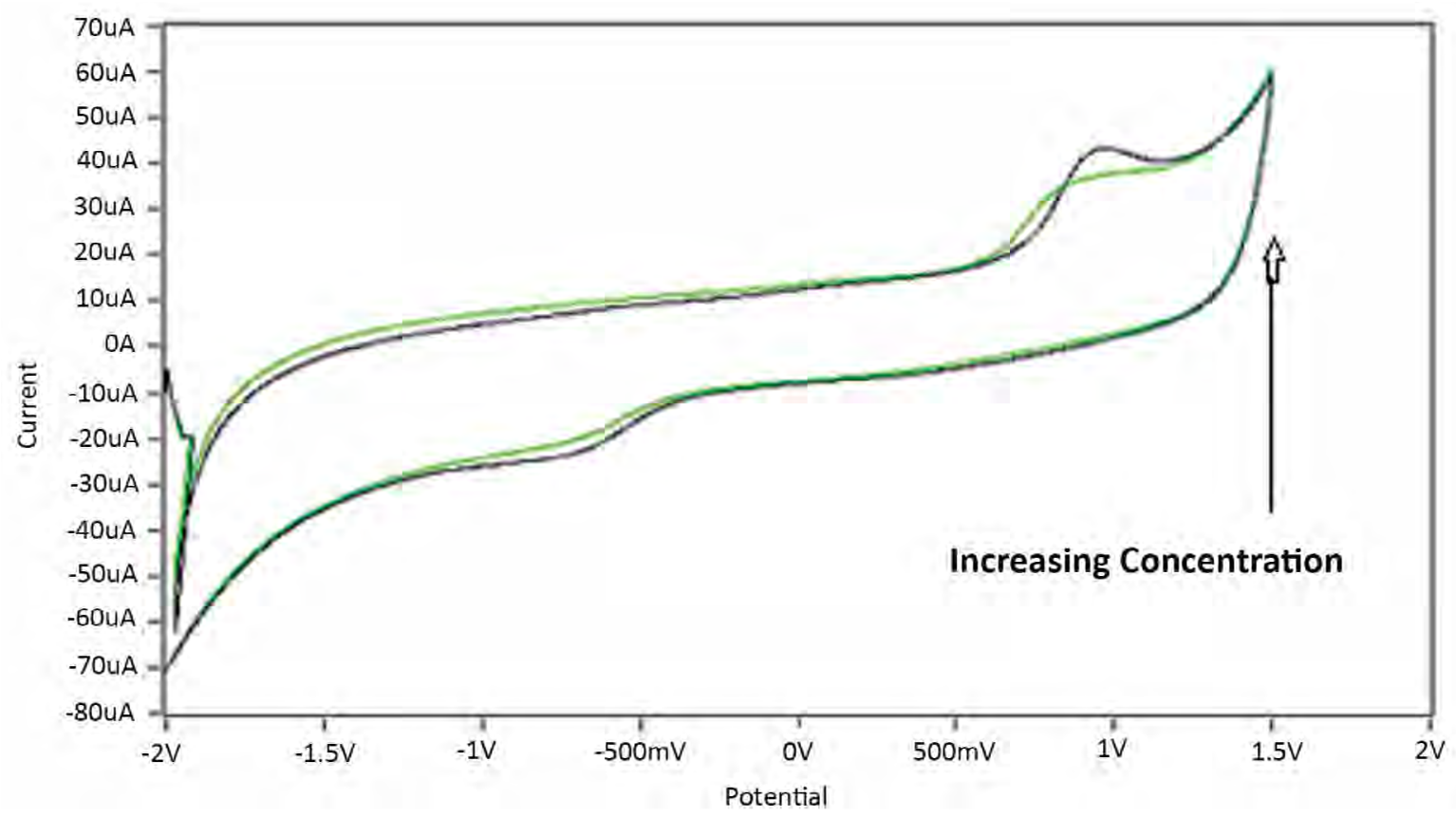

FIGURE 9. Cyclic voltammogram of different concentration of azithromycin nanoparticles in blood serum on $\mathrm{GCE}$ and $\mathrm{Ag} / \mathrm{AgCl}$ at $100 \mathrm{mVsec}-1$

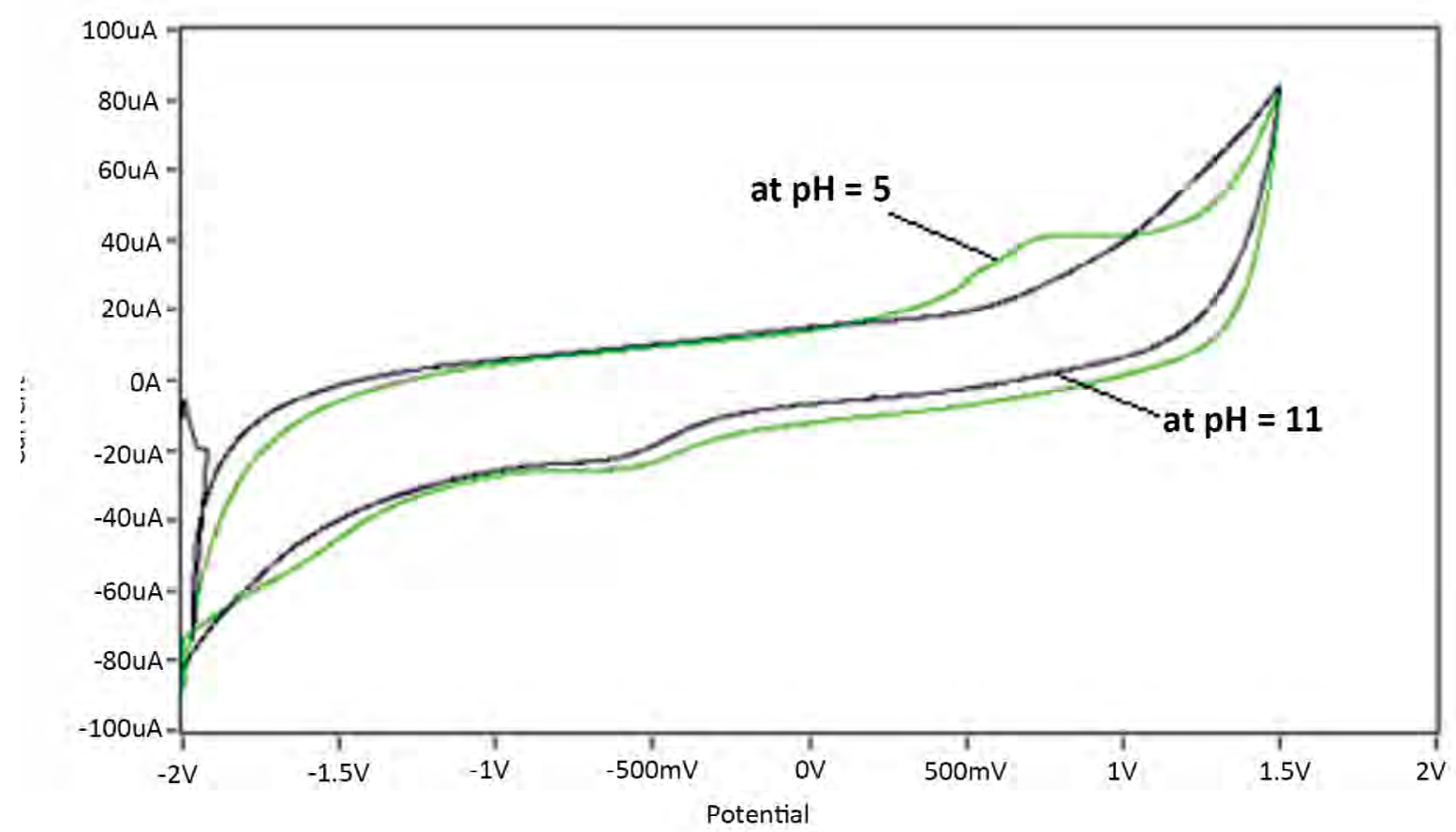

FIGURE 10. Cyclic voltammogram of azithromycin nanoparticles in blood medium at different pH (5 and 11) on GCE as working electrode and $\mathrm{Ag} / \mathrm{AgCl}$ as reference electrode, $100 \mathrm{mVsec}-1$

properties of current peaks of azithromycin nanoparticles in different $\mathrm{pH}$ of blood medium respectively.

\section{Effect of different scan rates}

Figure 13 illustrates the cyclic voltammogram of azithromycin nanoparticles in blood medium at different scan rates from 0.01-0.1 V/sec. the electrochemical properties of azithromycin nanoparticles in blood medium was enhanced the oxida- tion-reduction current peaks against to gradually increasing the scan rate.

\section{Reliability and stability study}

Azithromycin nanoparticles compound in blood medium was studied by GCE as a sensor in cyclic voltammetry. To ensure that the results were reliable in this study, ten times of scanning was used for the voltammogram was shown in Figure 14, and 


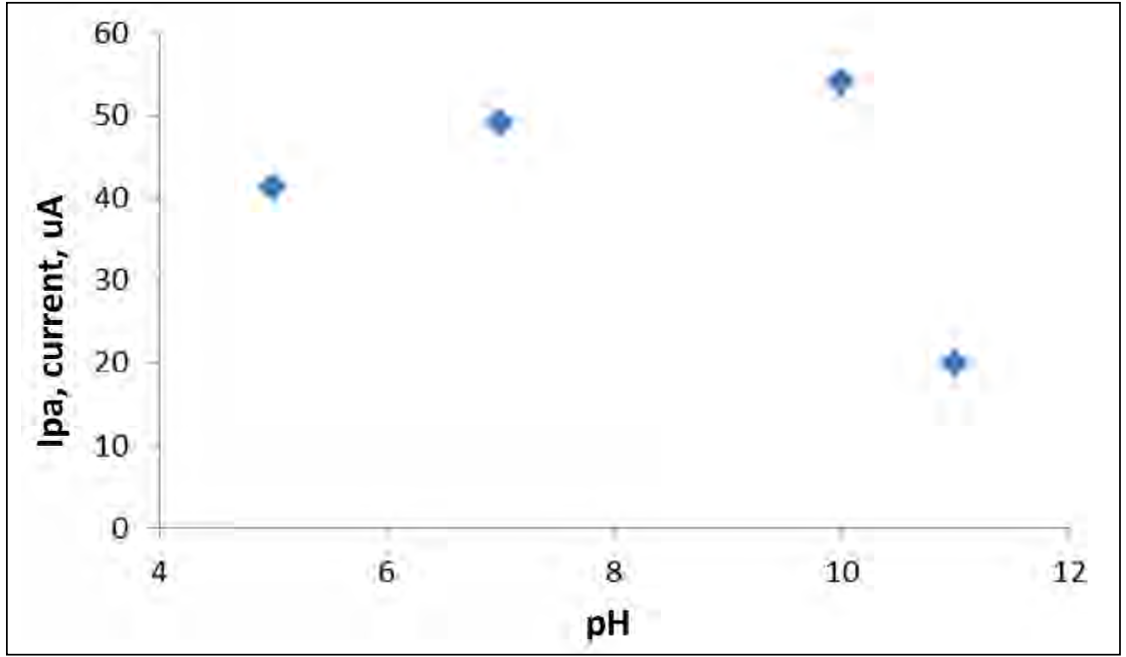

FIGURE 11. Relationship between different $\mathrm{pH}$ and oxidation current peaks of azithromycin nanoparticles in blood medium

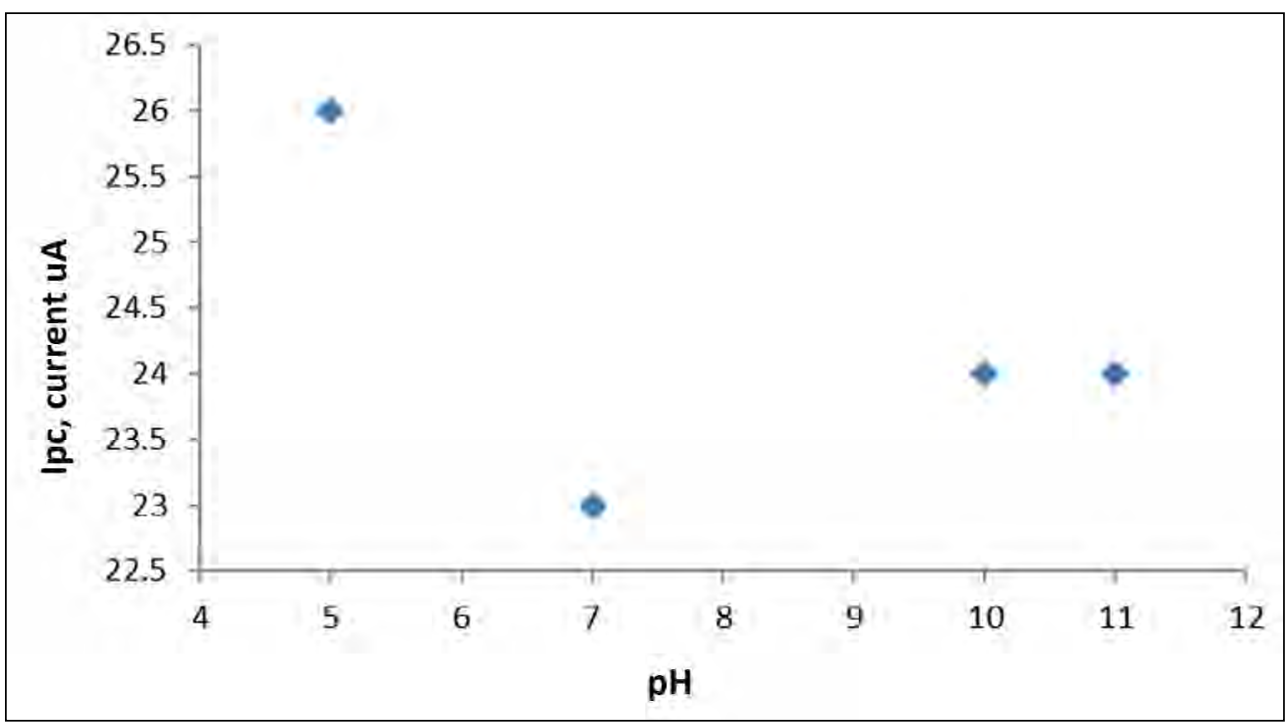

FIGURE 12. Relationship between different $\mathrm{pH}$ and reducation current peaks of azithromycin nanoparticles in blood medium

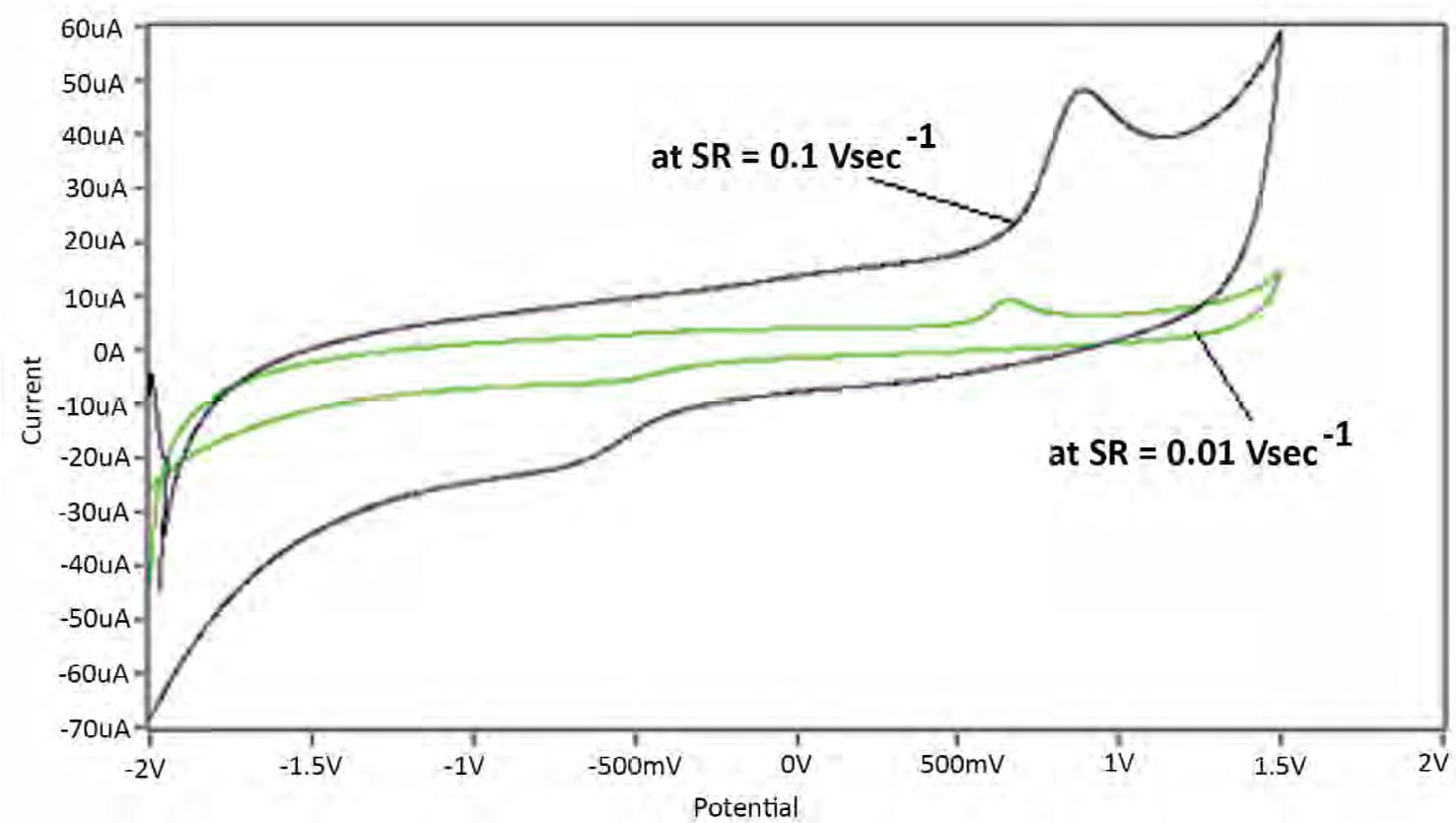

FIGURE 13. Cyclic voltammogram of azithromycin nanoparticles in blood medium at different scan rate (0.01-0.1 Vsec $\left.^{-1}\right)$ 


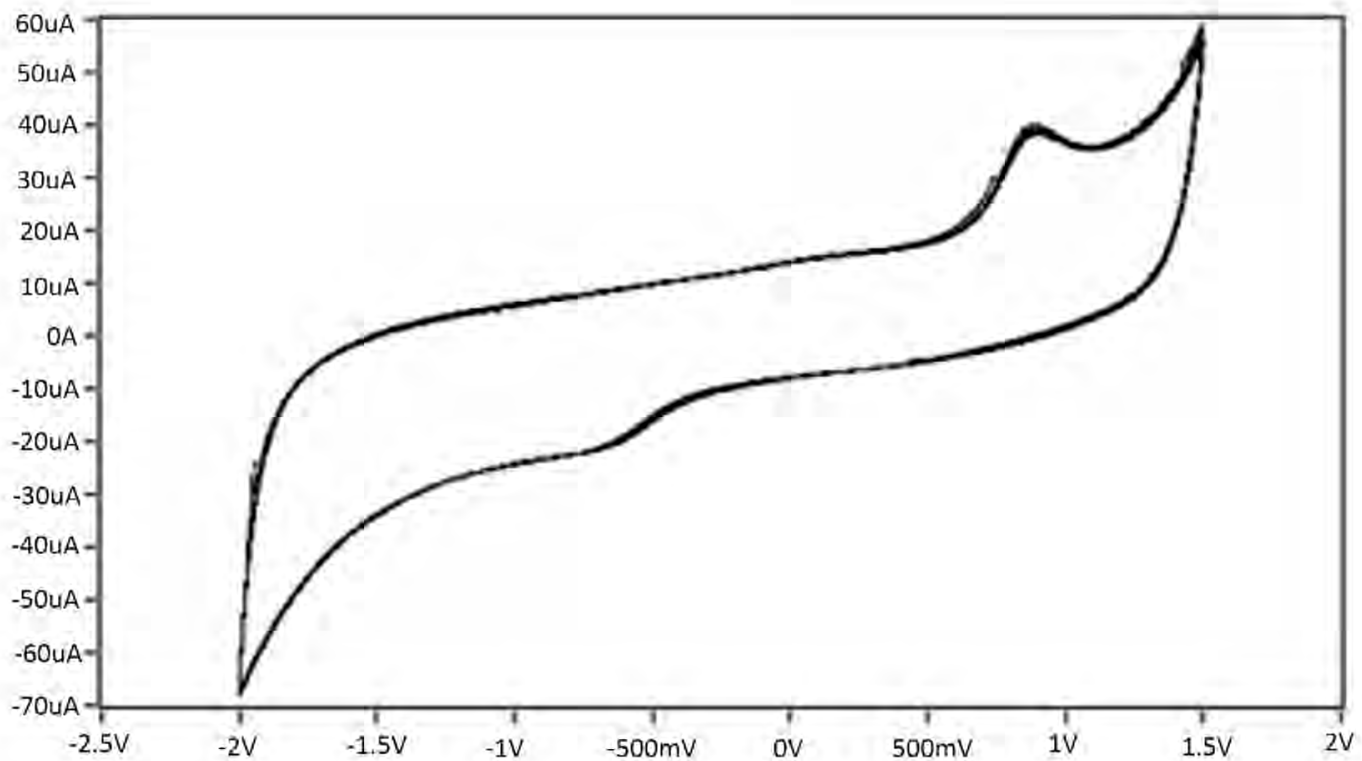

FIGURE 14. Cyclic voltammogram of azithromycin nanoparticles in blood medium at ten times recording

the relative standard deviation (RSD) was determined for both oxidation-reduction current peaks of azithromycin nanoparticles $\pm 0.329 \%$ and \pm $0.411 \%$, respectively [14].

\section{CONCLUSIONS}

Azithromycin compound was converted into nanoparticles and studied by cyclic voltammetric technique to find the electrochemical behavior in blood medium at different $\mathrm{pH}$, scan rates and study

\section{REFERENCES}

1. Radhi MM, Al-Damlooji NK, Abed BK et al. Electrochemical sensors for detecting Mn (II) in blood medium. Sensors \& Transducers, 2013; 149(2):89-93.

2. Radhi MM, Abdullah $\mathrm{HN}, \mathrm{Al}-$ Asadi $\mathrm{SA}$ et al. Electrochemical oxidation effect of ascorbic acid on mercury ions in blood sample using cyclic voltammetry. Int J Ind Chem. 2015;6(4):311-316.

3. Radhi MM, Tan WT. Voltammetric detection of Mn(II) in blood sample at $\mathrm{C} 60$ and MWCNT modified glassy carbon electrodes. Ame J Appli. Sci. 2010;7(3):439-445.

4. Radhi MM, Dawood DS, Al-Damlooji NK. Development of electrochemical sensors for the detectionof mercury by CNT/Li+ , $\mathrm{C} 60 / \mathrm{Li}+$ and activated carbon modified glassy carbon electrode in blood medium. Sensors \& Transdu. 2012;146(11):191-202.

5. Radhi MM, Khalaf MZ, Ali ZO et al. Voltammetric analysis of Zn (II) in present of each ascorbic acid (AA) and folic acid (FA) in human blood samples. AASCIT Commus, 2016;3:113-119.

6. Greenwood, David. Antimicrobial drugs: Chronicle of a twentieth century medical triumph (1st ed.). Oxford University Press, 2008, p. 239.

7. Wu Y1, Ji X, Hu S. Studies on electrochemical oxidation of azithromycin and its interaction with bovine serum albumin. Bioelectrochemistry. 2004 Aug;64(1):91-7.

8. Jafari S, Dehghani M, Nasirizadeh N, Azimzadeh M. An azithromycin electrochemical sensor based on an aniline MIP film electropolymerized the reliability - stability of the nanoparticles in blood medium. It was found that azithromycin nanoparticles can be considered as antioxidative antibiotic reagent in alkaline blood serum medium which showed two cathodic current peaks to appear and the oxidation current peak was disappeared in the cyclic voltammogram. The study was indicated that azithromycin nanoparticles compound in both micro and nanoparticles are good antioxidant antibiotic reagents in alkaline blood medium.

Conflict of interest: none declared Financial support: none declared

on a gold nano urchins/graphene oxide modified glassy carbon electrode. Journal of Electroanalytical Chemistry 2018; 829:27-34.

9. Christopher MA. Oliveira-Brett AM. Bioelectroanalysis of pharmaceutical compounds. Bioanal Rev. 2012;4:31-53.

10. Abdelwahed W, Degobert G, Stainmesse S, Fessi H. Freeze-drying of nanoparticles: formulation, process and storage considerations. Adv Drug Deliv Rev. 2006 Dec 30;58(15):1688-713.

11. Muhammed Mizher Radhi, Anfal Ismael Ibrahim, Yousif Kadhim Al-Haidarie, Sura Ali Al-Asadi, Emad Abbas Jaffar Al-Mulla, Rifampicin: Electrochemical Effect on Blood Component by Cyclic Voltammetry Using NanoSensor. Nano Biomed. Eng. 2019;11(2):150-156.

12. Sochor J, Dobes J, Krystofova $O$ et al. Electrochemistry as a Tool for Studying Antioxidant Properties. International Journal of Electrochemical Science, 2013;8:8464-8489.

13. Muhammed Mizher Radhi, Intesar Nadhum Khelkal, Eman Natiq Naji, Alaa Laebi Abdullah. Electrochemical characterization of micro- and nano-particles of ceftriaxone in human blood serum samples using cyclic voltammetry. Journal of Silicate Based and Composite Materials, 2019;71(2):54-58.

14. Engin C, Yilmaz S, Saglikoglu G, Yagmur S, Sadikoglu M. Electroanalytical Investigation of Paracetamol on Glassy Carbon Electrode by Voltammetry. International Journal of Electrochemical Science, 2015;10:1916-1925. 